\title{
Sistemas alimentarios globales $y$ ley de etiquetado de alimentos en Chile
}

\author{
Maycon Noremberg Schubert \\ Universidade Federal do Rio Grande do Sul - Porto Alegre - RS - Brasil \\ ORCID: https://orcid.org/0000-0003-3699-525X \\ David Eduardo Ávalos \\ Universidade Federal do Rio Grande do Sul - Porto Alegre - RS - Brasil \\ ORCID: https://orcid.org/0000-0002-2367-6973
}

\section{Resumen}

El presente trabajo se propone analizar el papel del gobierno chileno frente al modelo de producción corporativa de alimentos, evaluando la política pública de rotulado que desarrolló el Ministerio de Salud en los últimos años a través de la ley 20.606. Se estudia el impacto de la ley de rotulado de alimentos en los cambios en los patrones de consumo global, así como sus resultados recientes en Chile. En el contexto actual, el Estado pierde importancia como regulador de la producción y consumo alimentario en el mundo, lo que hace que el régimen corporativista tenga mayor libertad para entrar en la dieta de los hogares. Se trabaja a partir de la economía política agraria de los regímenes alimentarios, del rol del Estado en la regulación del consumo alimentar, así como del debate reciente en torno a la obesidad. Se analizan estudios nacionales e internacionales que miden el impacto de la aplicación de políticas gubernamentales de etiquetado de alimentos en la reducción o contención de los niveles de sobrepeso u obesidad. Se descubre que los mecanismos de rotulado aplicados llevan a una reducción general en el consumo de productos ultraprocesados, y ayudan a que las industrias incorporen más nutrientes a sus alimentos. No obstante, no se logra erradicar su consumo debido a que la población con menos ingresos no dispone de mecanismos eficientes para reemplazarlos. Además, políticas complementarias son necesarias, como incentivos a los mercados alternativos y con subsidios e impuestos sobre los alimentos ultraprocesados.

Palabras clave: Sistemas alimentarios. Obesidad. Ley de etiquetado. Consumo.

\section{Sistemas alimentares globais e lei de rotulagem de alimentos no Chile}

\section{Resumo}

Esse trabalho tem como objetivo analisar o papel do governo chileno frente ao modelo de produção corporativa de alimentos, avaliando a política pública de rotulagem desenvolvida pelo Ministério da Saúde nos últimos anos através da Lei 20.606. Apresentam-se alguns resultados de leis de rotulagem de alimentos sobre as mudanças nos padrões de consumo, assim como seus primeiros resultados no Chile. No contexto atual, o Estado perde 
importância como regulador da produção e consumo de alimentos no mundo, o que faz com que o regime corporativo tenha mais liberdade para entrar na dieta familiar. O trabalho baseia-se na abordagem dos regimes alimentares para discutir o papel do Estado na regulação do consumo alimentar. São analisados estudos nacionais e internacionais que medem o impacto da implementação de políticas governamentais de rotulagem de alimentos na redução ou contenção dos níveis de excesso de peso ou obesidade. Verifica-se que, embora os mecanismos de rotulagem conduzam a uma redução global do consumo de produtos ultra processados e ajudem as indústrias a incorporar mais nutrientes nos seus alimentos, o seu consumo não é erradicado porque a população com menos renda não tem melhores alternativas para substituí-los. Ademais, políticas complementares são necessárias, como incentivos aos mercados alternativos, e com subsídios e impostos sobre os alimentos ultraprocessados.

Palavras-chave: Sistemas alimentares. Obesidade. Lei de rotulagem. Consumo.

\section{Abstract}

\section{Global food systems and food labeling law in Chile}

This paper aims to analyze the role of the Chilean government in relation to the corporate food production model, evaluating the public policy of labeling developed by the Ministry of Health in recent years through Law 20.606. It studies the impact of the food labeling law on changes in global consumption patterns, as well as its first results in Chile. In the current context, the State is losing importance as a regulator of food production and consumption in the world, which means that the corporatist regime has greater freedom to enter the household diet. The work is based on the theoretical tradition of food regimes, the role of the State in regulating food consumption, and the recent debate on obesity. In this paper, we review national and international studies that measure the impact of the implementation of government food labeling policies on the reduction or maintenance of overweight or obesity levels. It finds that while labeling mechanisms lead to an overall reduction in consumption of ultraprocessed products, and help industries to incorporate more nutrients into their foods. Nevertheless, their consumption is not eradicated because lower income populations do not have better alternatives to replace them. In addition, complementary policies are necessary, as an incentive to alternative markets and with subsidies and taxes on ultra-processed foods.

Keywords: Food systems. Obesity. Labeling law. Consumption.

\section{Introducción: La insalubridad alimentaria como problemática en el mundo y en Chile}

La consolidación de las corporaciones de producción y consumo de alimentos (entre ellas, las cadenas de supermercado) ha llevado a la explosión de la oferta de productos ultraprocesados en el mundo. Estos conglomerados internacionales sustituyen el modelo alimentario que se basaba en el consumo de productos elaborados por las mismas personas dentro del hogar, adaptándose a ciertos cambios sociales (entrada de la mujer al mercado del trabajo, reducción en los tiempos para preparar alimentos, entre otras), haciendo disponibles productos listos para consumir, ricos en energía y escasos en nutrientes. Este cambio de paradigma se justifica bajo la premisa de que el aumento en la disponibilidad de productos acabaría con el hambre y la pobreza, lo que en la práctica no se consiguió (PREISS y MARQUES, 2015).

Desde el punto de vista de la producción alimentar, se ha analizado la diferencia entre países centrales y periféricos a nivel mundial, para indicar que la 
oferta de ultraprocesados se realiza no sólo con el fin de generar mayores excedentes, sino también para que las corporaciones puedan ir entrando en los diversos países a través de la oferta de productos, expandiendo su influencia y poder de mercado a nivel internacional (OOSTERVER y SONNNENFELD, 2012). Paralelo a estos cambios ocurre el proceso, que ya fue conocido en el trabajo de Marx, de mercantilización de la economía, el cual indica la importancia del valor de cambio de los productos, por sobre el valor de uso, y en donde se forma la cadena mundial de productos básicos (FRIEDMANN, 2016).

En cuanto al consumo, y tomando como base lo ocurrido en la sociedad francesa del siglo XX, existió una desigualdad que no sólo se expresó en el mercado de trabajo, sino también en la degradación de la alimentación de las personas, con lo cual las clases precarizadas ven deteriorada su salud, ya que consumen menos frutas, legumbres, peces y carnes. En cambio, estas poblaciones son más susceptibles de consumir bebidas azucaradas, tener una alimentación menos diversificada - que se manifiesta en el consumo de platos únicos - experimentar la comida fuera de hora, y con altos niveles de sedentarismo. Todas estas condiciones son factores que generan una propensión a desarrollar la obesidad en aquellas poblaciones más precarizadas (POULAIN, 2014).

Diversos estudios demuestran una asociación entre el consumo de determinado tipo de alimentos y el nivel socioeconómico, siendo los ultraprocesados mayormente servidos por los sectores con ingresos económicos bajos (AOUNALLAH-SKHIRI et al., 2011; BIBLIONI et al., 2015; CRAIG et al., 2010; ARANEDA et al., 2016). La obesidad se ha acrecentado a lo largo del tiempo y el espacio, siendo inicialmente problemática en los países de altos ingresos, seguida de su impacto a países de ingreso medio (MONTEIRO et al., 2013).

El panorama latinoamericano muestra que existe una alta prevalencia de la diabetes a nivel regional. Los datos del Bando Mundial (2019) indican que un 8,7\% de la población la padece, siendo más altos los niveles en México, Guyana, Nicaragua y Guatemala, los cuales superan el promedio de Latinoamérica. En cuanto a la prevalencia de sobrepeso en la población adulta, 6 de cada 10 personas lo experimentan en la región. México y Chile son los países con los mayores niveles en América Latina, con un aumento sostenido a lo largo de los últimos años. Mientras en México el sobrepeso aumentó del 55,9\% al 64,9\% entre 2000 y 2016 , en Chile este nivel pasó del 54,5\% al 63,1\% (BANCO MUNDIAL, 2019). Esta alta prevalencia del sobrepeso exhorta una reflexión profunda acerca del entorno alimentario de los ciudadanos en estos países, así como buscar entender cuál es el papel de los gobiernos para hacer frente a esta problemática social de salud.

Chile es el segundo país con el mayor consumo de bebidas azucaradas y uno de los líderes en la ingesta de 'comida chatarra' o alimentos ultraprocesados. El gobierno chileno ha implementado varios mecanismos de control al consumo de estos productos, como los impuestos a las bebidas listas para servir, así como el etiquetado de alimentos para fomentar la compra de productos más saludables (CARO et al., 2018). Estos mecanismos se han realizado en los últimos años con el fin de mejorar la calidad de vida de la población chilena, permitiendo que dispongan de información más simple de entender en los envases al momento de considerar la compra de un alimento. 
En el caso de la realidad chilena existe un conjunto de datos preocupantes, ya que el $67 \%$ de la población muestra algún grado de sobrepeso, con un 39,9\% que posee un sobrepeso leve, un $25,1 \%$ es obeso y un $2,3 \%$ tiene obesidad mórbida. De esta manera, existe un gasto en los sistemas de salud vinculados a la obesidad, como pensiones de invalidez, ausentismo laboral, pérdida de productividad, entre otros, y por tanto a partir de este diagnóstico se justificaría el uso de políticas públicas para combatirla (SÁNCHEZ y SILVA, 2017, p. 9). Un informe de OPS (2015) que se basa en los datos de Euromonitor internacional (2014), da cuenta de que Chile es uno de los países con mayor consumo per cápita de alimentos ultraprocesados, ocupando el séptimo lugar de un listado de 80 países analizados, y siendo sólo superado por México a nivel latinoamericano.

Este trabajo responde a la necesidad de estudiar las políticas públicas de etiquetado o rotulado de alimentos considerando tanto los sistemas como los regímenes alimentarios, el papel del Estado y los diversos actores que son parte de este modelo de producción a través del estudio de sus formas de consumo.

\section{Sistemas y regímenes alimentarios}

Una primera noción clave para entender los cambios recientes en las dinámicas alimenticias es el concepto de sistemas alimentarios. Éstos son modelos que tienen un funcionamiento interno y que toman elementos del entorno global, configurando un tipo específico de producción. En este contexto, estos sistemas socioeconómicos globales se relacionan de manera problemática con el ambiente, al entender que éstos tienen un impacto en una dimensión global, como la intensificación de la agricultura, concentración/fragmentación de la propiedad mundial, concentración corporativa y mercados globalizados que tensiona la relación con el ambiente (ERICKSON, 2008).

Desde una perspectiva en donde se vincula salud del cuerpo y del planeta, un documento escrito por el Programa de las Naciones Unidas para el Desarrollo (UNEP, 2016) diagnostica que los sistemas alimentarios han generado impactos tanto en la agricultura como en los patrones de consumo. Por una parte, causan daños en la tierra, degradándola y haciendo perder sus nutrientes, con efectos sobre la biodiversidad terrestre y acuática. En tanto que desde la salud humana se produce el cambio de dietas hacia un uso alimentar insalubre e insostenible. De esta manera, el diagnóstico en torno al impacto de los sistemas alimentarios conlleva también la elaboración de propuestas, que pasan por mejorar la eficiencia de la producción. Así, son interesantes las decisiones basadas en La Agenda 2030 para el Desarrollo Sostenible, la cual procura combatir la pobreza y el hambre, y a su vez busca reducir los niveles de obesidad en el planeta a partir de soluciones sistémicas (UNEP, 2016). Estas posturas sistémicas analizan el fenómeno alimentar desde una perspectiva estructural, al centrarse en la relación entre el cambio ambiental y la producción agrícola como causas globales que generan una diversidad de problemas para los ecosistemas e individuos, quienes se encuentran impedidos de ejercer su capacidad de agencia.

En un sentido distinto a la concepción de sistemas alimentarios indicada arriba, se argumenta que los actores tradicionales no perdieron su capacidad de acción política (Estado, sindicatos, partidos, etc.). Existen alianzas entre nuevos 
actores, que construyen demandas políticas, como la del movimiento ambientalista con el movimiento de defensa del consumidor. Desde esta perspectiva, entonces, los consumidores no son actores que van en dirección libre hacia la sustentabilidad; tampoco son víctimas pasivas y manipuladas de las formas dominantes de producción, es decir, son una mezcla de agencia y estructura (PORTILHO, 2010). Esta visión va en la misma línea de quien indica que el consumo se vuelve la forma de expresión ciudadana, y por lo tanto hoy en día las identidades políticas se definen por procesos históricos sustentados en el consumo (CANCLINI, 1997). Otra postura que vincula el nivel de agencia-estructura es la que realizan quienes entienden que el cambio en los sistemas alimentarios se da tanto a nivel estructural, con la transición hacia un modelo agroecológico, como a nivel individual, con el paso hacia la agricultura orgánica (CARON, 2018). Sin embargo, otras posturas señalan que las estrategias de circuitos alternativos no necesariamente son sustentables, ya que pueden mantener en vez de anular las desigualdades económicas y ambientales existentes (TREGEAR, 2011).

Otro abordaje teórico-metodológico es el de los regímenes alimentarios, que indica que dinámica actual de expansión del sistema agroalimentario global es una continuación a la Revolución Verde, que conduce al aumento de los rendimientos de los cereales, se intensifica la agricultura a partir de modificaciones tecnológicas, aumenta la concentración de la tierra y se introduce el uso de fertilizantes y pesticidas (HOLT GIMÉNEZ, 2011; MCMICHAEL, 2016). Ambos autores hacen alusión al tercer régimen alimentario corporativista, el que se sitúa en los años setenta y ochenta del siglo pasado, y que nace paralelamente a los cambios en la economía hacia un modelo capitalista neoliberal. Estos cambios se sustentan principalmente a partir del desmantelamiento de las juntas nacionales de comercialización, y de la destrucción de la investigación agrícola nacional. Estas políticas se integraron en tratados internacionales a través de acuerdos bilaterales e internacionales de libre comercio (TLC). Tales acuerdos permiten que avance el tercer régimen alimentario en aquellos países que suscribieron estos tratados, cristalizados en la creación de la Organización Mundial de Comercio. A través de ésta, se institucionaliza el proceso de liberalización agrícola en una escala global, y los Estados pierden la capacidad de regular la alimentación y la agricultura (HOLT GIMÉNEZ, 2011).

El método que lleva implícito el análisis de los regímenes alimentarios entiende a las corporaciones sin una referencia territorial y social específica, con lo cual suprime la pregunta por la diversidad de cultivos, por los tipos de agricultores, así como por los flujos de productos o mercancías. Con ello, el análisis estructural suprime ciertas dinámicas que existen entre las corporaciones, Estado y movimientos sociales, por ejemplo, los procesos de aprendizaje que tienen las corporaciones en torno a los movimientos sociales, incorporando conceptos provenientes de la crítica de estos últimos a los primeros (FRIEDMANN, 2016).

Pese a estas críticas, este método es capaz de dar cuenta de la pérdida de derechos de regulación nacional, lo cual permite entender los procesos de imposición de productos alimenticios en el mundo, con repercusiones en Latinoamérica y Chile, lo que después llevará a la necesidad de leyes de etiquetado como formas de gobernanza frente a estos mecanismos globales de desregulación alimentar en favor de un modelo industrial. 


\section{El sistema agroalimentario de Chile}

Los orígenes de la modernización de la agricultura en Chile se encuentran en los procesos de reforma y contrarreforma agraria sustentados inicialmente bajo los gobiernos de Frei y Allende, los cuales fueron sólo parcialmente contrarrestados durante la dictadura de Pinochet, ya que sólo una parte minoritaria de las tierras fueron devueltas a los antiguos dueños, siendo otra gran proporción asignada a productores no sindicalizados y que no militaran en partidos políticos de izquierda (BELLISARIO, 2007; 2013; PANEZ et al., 2020).

El paso al tercer régimen alimentario en Chile ocurrió desde mediados de la década de los setenta, siguiendo la dinámica de los países del hemisferio sur, los cuales comienzan a tener un rol importante en el suministro de productos de exportación de bajo consumo interno y alto precio, como frutas fuera de temporada, hortalizas y flores (RÍOS-NÚÑEZ, 2013) y por lo tanto se privilegió la explotación de los recursos naturales para ser vendidos en mercados globales (BELLISARIO, 2013). Bajo la dictadura militar, la mayor parte de los mercados de productos e insumos fueron liberalizados, se termina con el latifundio, se consolidan las explotaciones de tamaño pequeño y mediano, se abre paso a un modelo sustentado en cultivos no-tradicionales, y se privatizan los derechos de agua (HOJMAN et al., 1990; CHANDLER, 2006; PANEZ et al., 2020). Una primera fase de este proceso abarca entre el año 1975 y 1982, en donde ocurre una serie de políticas públicas como la desgravación arancelaria, privatización de empresas públicas que eran parte de las cadenas agroalimentarias, reducción del empleo permanente en el sector, y el aumento del crédito a los productores (RíOS-NÚÑEZ, 2013).

Un segundo momento de este proceso lo constituye el periodo que va desde 1983 a 1997, y contempla el mayor poder de intervención del Estado en los asuntos de política monetaria, mediante el alza arancelaria, la intervención de mercados agrarios a través de bandas de precios a ciertos cultivos tradicionales, y el fomento a las exportaciones, lo que llevó al aumento sostenido de los envíos de fruta al extranjero en la década de los noventa (RÍOS-NÚÑEZ, 2013; PANEZ et al., 2020).

Otro planteamiento indica que la implementación del capitalismo agrario en Chile se realizó a través de la introducción de subsidios e incentivos para cultivos agrícolas como frutas, viñedos y plantaciones forestales de pinos y eucaliptos (BELLISARIO, 2013). Esta orientación político-económica fue cambiando el interés de los antiguos y nuevos productores del campo chileno, quienes se preocupan cada vez menos de abastecer a los mercados locales, y constituyen empresas o corporaciones orientadas a la exportación de alimentos.

De este modo, durante la década de los noventa, se produce un crecimiento de la producción agroindustrial, en donde se intensifica el papel del capital en los procesos de producción, circulación, comercialización y consumo; junto a ello se sustituyen los productos agrícolas por los agroindustriales, emergen oligopolios que concentran la producción orientada hacia el consumidor mundial, se re-concentran las tierras, y aumentan los monocultivos gracias a la intensificación del uso de energía, agua, suelos y agroquímicos (PANEZ et al., 2020). Estas dinámicas fueron facilitadas por el Estado a través de la entrega de subsidios y créditos para la construcción de infraestructura que facilita la productividad (apoyo técnico al riego) así como a la distribución de productos (construcción o mejora de carreteras). La 
firma de tratados de libre comercio fue el paso siguiente para la institucionalización del modelo de apertura internacional que facilitó la salida y entrada de productos a Chile, abriéndose al proceso de globalización alimentaria, en donde comienzan a importarse productos fabricados industrialmente y a exportar principalmente frutas.

Por el lado de las importaciones, la sobreoferta de productos alimenticios procesados y ultraprocesados fue un fenómeno que comienza a aparecer en Chile en las últimas décadas del siglo XX. En particular, la crisis económica que se extendió entre 1982 y 1986 hizo cambiar los patrones alimenticios, aumentando el consumo de grasas animales y vegetales y disminuyendo la ingesta de cereales, vegetales y frutas hacia principios del siglo XXI (UAUY y VIO, 2007). Para Mendoza et al. (2007) entre 1988 y 1997 aumenta el consumo de grasas en un 50,5\%, y se incrementa en un 31,1\% el gasto en alimentación, razón por la cual atribuyen el aumento en el cambio de productos consumidos a factores económicos y sociodemográficos. Otros autores han definido los cambios en la disponibilidad de producción como el paso hacia una dieta "occidental" en donde al consumo de grasas y comida rápida se le suma el incremento del sedentarismo, lo que lleva a aumentar los niveles de presión sanguínea en la población adulta (ALBALA et al., 2002). Estos enfoques explican los cambios alimentarios de principio de la década de los noventa atendiendo a las características personales, como variaciones en la dieta y el creciente sedentarismo que llevan a la aparición de la obesidad, el aumento del colesterol en la sangre, la diabetes tipo 2, o la hipertensión (ALBALA et al., 2002). Bajo este enfoque, sería necesario mudar las características culturales y/o sicológicas de los chilenos para erradicar la alimentación escasamente nutritiva (pese a reconocer la existencia de factores externos, como por ejemplo las campañas publicitarias).

Desde una óptica más general, varios autores confirman el mayor ritmo de crecimiento del consumo de los productos de origen animal por sobre los de origen vegetal, lo que es producido por la menor disponibilidad de estos últimos productos para los ciudadanos chilenos. Tomando datos de las hojas de balance de la FAO, algunos planteamientos muestran que para inicios del siglo XXI disminuye la disponibilidad de frutas, pasando de $60,6 \mathrm{~kg}$ por año por persona a 47,2 kg en 2003, y aumenta la presencia de leguminosas, cereales, aceites, azúcares y endulzantes en el mismo periodo de tiempo. En lo que respecta a productos de origen animal, hay un incremento en la disponibilidad de carnes, así como una reducción en la oferta de pescados y mariscos entre 1995 y 2003. Mendoza et al. (2007) concluyen que la baja en la disponibilidad de los alimentos sanos no se debe a que disminuye su producción a nivel nacional, sino porque aumentan las exportaciones, y esto hace reducir su disponibilidad interna. Es por ello que la disminución en la oferta de productos alimenticios sanos en el país obedece a problemas de distribución y no de producción. Ríos-Núñez (2013), argumenta que sumado a ello, la excesiva inequidad en la distribución de los ingresos hace que se desincentive la venta de productos agrícolas hacia los mercados locales, ya que a lo largo de las últimas décadas no ha mejorado el poder adquisitivo de los chilenos.

Estos planteamientos, que vinculan la problemática de la insalubridad alimentaria a factores externos al individuo, explican con mayor claridad la incidencia de los entornos alimentarios en las prácticas de consumo de los 
ciudadanos chilenos. En esta misma línea se sitúa el trabajo de Monteiro et al. (2017) quienes indican que hacia el año 2010 ha aumentado el suministro de alimentos ultraprocesados por parte de los países desarrollados, tendencia que se comienza a replicar incluso en países de ingresos medios, como Chile.

\section{El papel de las políticas alimentarias}

Los trabajos más recientes que han estudiado las políticas alimentarias la conceptualizan desde diversas perspectivas. Éstas son respuestas al sistema alimentario, e incorporan visiones tanto de la sociedad civil, empresas y el gobierno, actores que se desenvuelven desde una perspectiva multinivel. Así, existen instituciones a nivel local, nacional, regional e internacional, que son la base de estas políticas alimentarias pero que no son necesariamente ordenadas por el Estado, sino que diferentes grupos de interés disputan su configuración (LANG y BARLING, 2009). Así, se puede entender, por ejemplo, el rol que juega el gobierno de Chile al realizar las leyes de etiquetado, como forma de hacer frente a los cambios globales, respuestas que se elaboran a partir de las preocupaciones de los expertos y los movimientos sociales frente al predominio de un modelo de alimentación industrial implementado por las corporaciones alimentarias.

El papel del Estado en las políticas alimentarias para conseguir el desarrollo sustentable debe procurar vincular la seguridad alimentaria, salud humana, nutrición, cambio climático y justicia social (CARON et al., 2018). Pese a ello, indican que, en la práctica, las políticas se enfocan en el mero suministro de alimentos, y en mecanismos para direccionar las externalidades negativas del modelo, razón por la cual se trataría - de un enfoque reformista (HOLT-GIMÉNEZ, 2011). Es así que diversos autores indican que los sistemas alimentarios deben promover la resiliencia, garantizar la seguridad alimentaria y nutricional de las personas, así como contribuir a la reducción de desigualdades, entre otros desafíos (CARON et al., 2018).

Como ejemplo, en el plano institucional, el Programa de las Naciones Unidas propone una serie de medidas de mitigación que podrían ser realizadas por los gobiernos, las empresas y la sociedad civil. Entre ellas se puede destacar el rol del gobierno educando en temáticas nutricionales a la población en general, las empresas facilitando las elecciones de alimentos saludables y sostenibles para los consumidores, y los agricultores suministrando alimentos (UNEP, 2016). Sin embargo, este enfoque entrega una mayor importancia a la disponibilidad de productos en los entornos urbanos para los consumidores, ignorando otras problemáticas como los desiertos alimentarios ofrecidos por el modelo corporativista.

Desde una perspectiva inercial, otros teóricos analizan la transición entre el papel del Estado como regulador de la alimentación hacia un nuevo paradigma, en donde los actores locales tienen más relación con redes transnacionales en todos los procesos de producción y consumo (OOSTERVER y SONNENFELD, 2012, p. 63). Citando como caso la creación de la WTO, los autores indican que los Estados pierden la capacidad de imponer barreras al flujo de alimentos y se configura el régimen global de alimentación. Esto también hace aparecer la necesidad de gobernanza por parte de organizaciones de la sociedad civil, ONGs y grupos de 
consumo, quienes han promovido mecanismos de etiquetado de productos, y por tanto van teniendo un papel importante como grupos sociales que contribuyen al diseño e implementación de políticas públicas alimentarias por parte de los Estados.

\section{La ley de etiquetado de alimentos como política en Chile}

El consumo de alimentos ultraprocesados es parte mayoritaria de la dieta de la población chilena, lo que ha generado una de las mayores tasas de obesidad en el mundo. Con el paso de los años, la población chilena ha tenido cada vez un mayor cuestionamiento a esta lógica de alimentación, lo que ha llevado a que diversos representantes del poder público y profesionales de la salud generen una propuesta tendiente a informar a la población, así como en mejorar el acceso a alimentos más saludables. Este proceso ha derivado en la creación el año 2016 de la ley etiquetado de alimentos (Ley 20.606), la que se origina en el proyecto de ley sobre 'regulación de alimentos poco saludables' presentado en el senado el 21 de marzo de 2007 (SÁNCHEZ y SILVA, 2017).

Esta ley promueve la entrega de información detallada de los ingredientes que contienen los productos, así como su información nutricional, siendo el Ministerio de Salud de Chile quien se encarga de elaborar las etiquetas de los productos, las cuales deben incluir los contenidos de energía, azúcares, sodio y grasas saturadas (MINSAL, 2016). A través de la Ley 20.606, el gobierno chileno intervino en el etiquetado, en las regulaciones a los mensajes publicitarios, así como en la restricción a la venta de alimentos en el interior y en los alrededores de escuelas. Pero hay una tensión entre el gobierno de Chile y las corporaciones a raíz de la implementación de estas políticas (SÁNCHEZ y SILVA, 2017). Para las autoras, el proyecto intenta cambiar los hábitos alimenticios con el fin de mejorar la salud y calidad de vida de la población. Este proyecto se centra en orientar la decisión del consumidor informando los ingredientes que tiene un producto y su valor nutricional, brindándole la posibilidad de compararlo con uno de otra marca. Esta visión desde la política pública se relaciona con el fenómeno de que el consumidor adquiere cada vez una mayor racionalidad, en tanto las decisiones de consumo son relevantes para la reproductibilidad política (CANCLINI, 1997).

Tales medidas propuestas fueron cuestionadas por la industria de alimentos, alegando que la ley no se basa en evidencia científica, ni se ha reportado tener efectos exitosos; se estigmatizan los alimentos procesados, los cuales no son mayoritariamente consumidos por los chilenos; se desincentiva la innovación o investigación en la industria alimentaria; se vulnera la propiedad intelectual y los derechos de marca; se generan barreras técnicas al comercio (SÁNCHEZ y SILVA, 2017).

Al hablar de los efectos del etiquetado de alimentos, Sánchez y Silva (2017) indican que un estudio realizado por In Situ y encargado por Chilealimentos muestra que, de una muestra acotada de población, el $41 \%$ declaró cambios en sus prácticas o hábitos alimenticios, reduciendo el consumo de productos con discos, reemplazándolos por bienes sustitutos con menos discos, u optando por alimentos sin discos. En la medición realizada por las autoras se estudiaron productos con tres, dos, uno y sin sellos de una empresa anónima de alimentos, en donde se indica que aquellos con sellos y que tienen sustitutos tienden a ser los más reemplazados, en 
tanto que aquellos alimentos sin sustitutos continúan teniendo un alto nivel de consumo; es por esta razón que las autoras indican que la ley permite mejorar las opciones de alimentación ya que vuelve más transparente e informado el mercado alimenticio. Sin embargo, se aprecia que el consumo de ultraprocesados continúa realizándose cuando no hay otros alimentos que los puedan reemplazar y que tengan costos similares (SÁNCHEZ y SILVA, 2017).

Resultados similares son apuntados por Santos-Antonio et al. (2019) sobre el impacto de la introducción de semáforos y rotulado de alimentos en diversos países. En primer lugar, se disminuye el consumo de alimentos altos en energía y bajos en nutrientes con el logo keyhole, hay inexistencia de cambios en los patrones de consumo con la introducción de logos Smart Choices y Choices. En otros casos, el etiquetado frontal de productos con grasas logró tener efectos tanto positivos como neutros en su consumo.

Otros factores como la presencia del color verde podrían incidir en su errónea comprensión, ya que se tendería a minimizar el impacto de los símbolos rojos o amarillos al encontrarse menos presentes en los productos para la realidad peruana (VALVERDE-AGUILAR et al., 2019). Entonces, en este país, la incorporación de semáforos tampoco ha permitido su completa comprensión al contar con un exceso de información a juicio de los autores, lo que ha ocurrido también para el caso de la realidad de Thailandia, en donde los semáforos han sido declarados como incomprensibles, siendo mejor evaluadas las etiquetas FoP, las cuales necesitan de menos campañas públicas complementarias (PONGUTTA et al., 2019). La ineficacia de estas políticas de etiquetado de alimentos en estos países son puestas en evidencia por los movimientos sociales, los cuales presionan a las corporaciones alimenticias para la entrega de información más clara en los envases de sus productos, y a su vez hacen un estricto seguimiento de los resultados de las políticas de etiquetado a través de los estudios de evaluación realizados por la comunidad científica. Es así como en Chile se ha implementado el símbolo octogonal que cataloga los diversos componentes de los productos industrializados como "alto en", descartándose la incorporación de semáforos-GDA (guideline daily amount) como mecanismos de entrega de información (VALVERDE-AGUILAR et al., 2019).

En otro estudio se analiza, desde una perspectiva cualitativa, las percepciones en torno al consumo alimentar en la población de Ecuador - que implementó el etiquetado de alimentos a productos procesados en 2014 a través de la incorporación de semáforos-GDA (FREIRE et al., 2017). Si bien los resultados no son generalizables a toda la población, el estudio evidencia que con la introducción del semáforo nutricional no cambian las prácticas en relación al consumo de alimentos procesados, teniendo efectos diferenciados según género, nivel socioeconómico, territorio y tramo etario (FREIRE et al., 2017).

Desde un punto de vista más general, las políticas de advertencia nutricional no se crean para generar cambios radicales en los patrones de consumo alimentario, sino más bien con el fin de incidir en la reformulación de los componentes de productos ultraprocesados, para hacerlos más saludables. Sin embargo, los autores comprobaron que, tras un año de puesta en marcha de la política de etiquetado, los consumidores adquirieron una mayor sensibilidad al considerarlo en sus decisiones de compra, y en paralelo no reportan cambios en la 
percepción de productos tradicionalmente poco saludables que incorporan en nutrientes positivos en sus productos, como es el caso de las salchichas (SCHNETTLER et al., 2019a). En otro estudio se indica que los individuos con un alto nivel de educación y mayores ingresos tienden a comprar alimentos más saludables y con menos contenidos de grasa y sal (SCHNETTLER et al., 2019b).

Pese a esta última evidencia, la mayor parte de estas mediciones indicadas precedentemente no exploran en profundidad en el impacto considerando la relación entre el nivel socioeconómico con el consumo de alimentos procesados y no procesados (artesanales). Una correcta caracterización del impacto de esta política pública vendría dada por el estudio de las tendencias de consumo de una alta variedad de alimentos procesados (y no sólo centrándose en algún producto en específico), así como por los niveles de sobrepeso u obesidad considerando a la población de ingresos altos, medios y bajos.

Así también se podrían estudiar nuevas variables que afectan a las políticas, por ejemplo, y siguiendo a Santos-Antonio et al. (2019) la información de etiquetado compite con otros factores como la presión de tiempo, y el conocimiento nutricional. Así, estos elementos individuales y contextuales limitan la efectividad del etiquetado, y es necesario de políticas complementarias que garanticen el acceso a los alimentos saludables, la promoción de la actividad física y la educación nutricional. Los estudios indicados en la siguiente sección muestran caminos alternativos a las políticas hasta ahora implementadas.

\section{Una exploración más allá de esta ley}

Existen muchos estudios que profundizan en la ineficacia de las medidas de etiquetado de productos como formas de cambio a nivel global de los patrones de alimentación en la sociedad actual.

En primer lugar, el combate al sobrepeso y la obesidad debe ir más allá de la existencia de estos mecanismos de rotulado, y debe estar acompañado por el aumento de los impuestos a otro grupo de alimentos adicionales así como a los ingredientes de las bebidas azucaradas (SSBs por sus siglas en inglés) como mecanismo de desincentivo al consumo de comida chatarra a nivel general, a través de la incidencia en sus precios, lo que además hace aumentar el presupuesto estatal disponible para ser invertido en los sistemas de salud (CARO, et al., 2017). Además, es necesaria la subvención a la compra de frutas, verduras y pescados, y la promoción del consumo de alimentos frescos y naturales a través de ferias libres y campañas en medios masivos.

En esta misma línea se sitúa el planteamiento de que si bien el gobierno de Chile ha realizado campañas para reducir el consumo de productos dañinos para la salud a través de su rotulado, no han existido mecanismos estatales que fomenten el consumo de alimentos altos en fibra o con menor contenido de colesterol (SCHNETTLER et al., 2019b). Es por ello que, en vez de centrarse en proporcionar una mayor información a la oferta de productos, la política debiera centrarse en bajar los costos de los alimentos altos en nutrientes producidos localmente.

En este sentido, las políticas alimentarias estatales no son suficientes para el combate al modelo de seguridad alimentaria propuesto por las grandes corporaciones. Estudios indican que hay que mirar los mecanismos a través de los 
cuales se configuran dinámicas que propician que sean las grandes corporaciones las que tengan derechos por sobre el de los Estados y ciudadanos. Para ello, se propone la visión de soberanía alimentaria para enfrentar el agotado modelo neoliberal, colocándola en el centro de diversos desafíos políticos, sociales, económicos y ecológicos (MCMICHAEL, 2016). Además, el régimen alimentar bajo el neoliberalismo institucionaliza la relación hegemónica donde los estados se subordinan al capital, específicamente a la Organización Mundial del Comercio (MCMICHAEL, 2016). Estas relaciones entre las instituciones estatales y los acuerdos internacionales de comercio mundial y los organismos que los sustentan, son base del estudio de que debe ser puesto en frente hoy como formas de combatir el problema del sobrepeso y obesidad en Chile y el mundo. Finalmente, las corporaciones pueden generar mecanismos de bloqueo a sus propios críticos, ya que toman los modelos alternativos al sistema convencional de producción de alimentos y los convierte en alimentos industriales, usando además las prácticas tradicionales de los propios agricultores (FRIEDMANN, 2016).

En Chile, sectores críticos a estos mecanismos de rotulado indican que no se cambia el juego de reglas impuesto por las corporaciones transnacionales. Actualmente el gobierno chileno está próximo a firmar el TPP 11, un tratado de comercialización entre 11 economías del pacífico. Notas de prensa recientes indican que la redacción del capítulo de propiedad intelectual del acuerdo fue escrito por las mismas empresas transnacionales, las cuales favorecen los intereses estadounidenses y perjudican a los chilenos (AKRAM, 2019). En el sector alimentario, esto se expresa en que las corporaciones transnacionales pueden demandar en tribunales internacionales a Chile, si es que no cumple con ciertas reglas, como por ejemplo la imposibilidad de diversificar la producción agrícola.

Si bien la ley de etiquetado informa sobre el contenido de los alimentos, y presiona hacia la adición de una mayor cantidad de nutrientes y fibra en los alimentos procesados, no propone otro modelo de alimentación distinto al régimen corporativista, lo cual hace que esta medida no tenga la efectividad suficiente para lograr cambios de base en los patrones alimentarios de la población chilena. El rotulado implementado por los gobiernos no ataca las desigualdades entre los modelos de producción corporativista, la pequeña producción y los consumidores más pobres del mundo, pese a que existe una tendencia generalizada hacia la reducción en el consumo de ultraprocesados (BERSTEIN y OYA, 2014).

Para el caso chileno, el rol de la política pública sólo se ha basado en la introducción de reformas sin atender la problemática alimenticia desde una óptica estructural, es decir, considerando la posibilidad de cambiar el tipo de producción hacia sistemas alimentarios que permitan aumentar la oferta de productos ambiental y socialmente sustentable y de mayor aporte nutricional.

\section{Conclusiones}

La realidad actual permite pensar en la ineficacia de los mecanismos de regulación estatal para hacer frente a modelos globales de alimentación que se imponen desde las corporaciones y bajo gobiernos reformistas. La experiencia internacional demuestra que el etiquetado, si bien tiene efectos en la reducción del consumo de alimentos 'altos en' no logra acabar con su total uso principalmente 
porque los sectores de menores ingresos económicos tienen una demanda casi inelástica por los ultraprocesados, en tanto que las corporaciones de alimentos sólo cambian parcialmente los componentes de sus productos con el fin de hacerlos más nutritivos. Así también, existe un problema más profundo para los consumidores, y es la escasa formación educacional para conocer los alimentos que les puedan aportar de mejor forma a su salud, así como su escaso poder de compra debido a su bajo nivel salarial.

En este contexto, cabe recordar que los Estados han perdido su importancia como entes reguladores de los sistemas mundiales de producción y consumo de alimentos. En gran medida, los Estados son incapaces de frenar las causas que estructuran la insalubridad alimentaria de las personas, ya que no logran hacer frente a la estructura diferenciada de precios que hace que los alimentos con menos nutrientes estén más al alcance de los sectores medios y pobres, en tanto que los alimentos de mayor valor nutricional están más presentes en las dietas de los grupos con mayores ingresos. La obesidad no sólo se produce por la disponibilidad de alimentos, sino que hay procesos de precarización de la clase obrera que se asocian a la presión a estas capas al consumo de estos productos ultraprocesados, siendo una precariedad objetiva que se manifiesta no sólo en los procesos de trabajo sino también en los espacios de consumo alimenticio fuera de lo laboral.

La incapacidad de los sectores de menores ingresos de poder acceder a alimentos saludables a un menor costo se produce por la existencia de una lógica de libre competencia que lleva a que los productores locales prefieran producir para los mercados con consumidores de mayor poder adquisitivo. Bajo esta lógica, y considerando la fuerte concentración de los ingresos en la sociedad chilena, no se ven grandes opciones para que la ciudadanía en su conjunto pueda alimentarse con productos nutritivos que sean una alternativa a los ultraprocesados. Es por ello que la problemática diagnosticada en este trabajo se relaciona con la imposibilidad que ofrece el modelo neoliberal de producción y distribución de alimentos para acabar con esta tendencia en la sociedad chilena.

Estas contradicciones de base socioeconómica son justamente propiciadas por acuerdos políticos institucionalizados del sistema global alimentario que sobrepasan las leyes nacionales, pero cuyas ideas se incrustan en las elites políticas, y que son las raíces de los modelos alimentarios que experimentan las poblaciones de Chile y el mundo. Así, es posible ver que los Estados tienen sólo un rol reformador de las políticas alimentarias en el contexto de dominio de las corporaciones internacionales en las dinámicas globales de producción, procesamiento y consumo de alimentos, siendo que su capacidad de incidencia es comprometida y dependiente a intereses corporativos, lo que limita procesos de cambio de la producción y el consumo hacia una de carácter más sustentable. En este sentido, son otros actores emergentes los que hoy en día tendrían la capacidad de afrontar las transformaciones del sistema alimentario, como los grupos de consumidores o agrupaciones de productores. Estos tienen un importante papel como generadores de una agenda alimentaria para las grandes corporaciones transnacionales y los Estados, así como proponen modelos alimenticios nutritivos al alcance de todos los ingresos monetarios y territorios, sin distinciones de clase social. 
Para el caso chileno, el panorama actual Ilama para la articulación de una estrategia de desarrollo territorial que promueva la disponibilidad de los alimentos más nutritivos en los mismos territorios rurales y sus articulaciones con las ciudades. La política pública, entonces, debe pasar por establecer iniciativas de apoyo a la producción local, no para insertarse como una competencia que exprese un modo de vida distinto al que promueve la producción industrial, sino que sea un modelo de producción y consumo que gradualmente vaya ganando un espacio en los territorios de Chile. De esta manera, la articulación de diversas estrategias de producción local puede sustentar la consecución de acuerdos que permitan crear nuevas normas que promuevan los intereses de los productores de alimentos nutritivos en relación a las leyes internacionales de comercio.

\section{REFERENCIAS}

AKRAM, H. Experto negociador contratado por el gobierno reconoció que texto del TPP fue dictado por transnacionales. In: CIPER Chile, 2019. Disponible en: https://ciperchile.cl/2019/08/12/experto-negociador-contratado-por-el-gobiernoreconocio-que-texto-del-tpp-fue-dictado-por-transnacionales Accedido en 15 de agosto 2019.

ALBALA, $C$. et al, Nutrition transition in Chile: determinants and consequences, Public health nutrition, v. 5, n. 1a, p. 123-128, 2002.

ARANEDA, J. et al. Consumo aparente de frutas, hortalizas y alimentos ultraprocesados en la población chilena. Revista chilena de nutrición, v. 43, n. 3, p. 271-278, 2016.

BANCO MUNDIAL. Obesity: Health and Economic Consequences of an Impending Global Challenge. In: The World Bank. Disponible en:

<https://www.worldbank.org/en/topic/nutrition/publication/obesity-health-andeconomic-consequences-of-an-impending-global-challenge> Accedido en 25 de febrero de 2019.

BELLISARIO, A, The Chilean Agrarian Transformation: Agrarian Reform and Capitalist 'Partial' Counter-Agrarian Reform, 1964-1980: Part 1: Reformism, Socialism and Free-Market Neoliberalism, Journal of Agrarian Change, v. 7, n. 1, p. 1-34, 2007.

BELLISARIO, A. La reforma agraria chilena. Reformismo, socialismo y neoliberalismo, 1964-1980., 2013.

BERNSTEIN, H.; OYA, C. Rural futures. How much should markets rule? International Institute for Environment and Development, October, 2014. Disponible en $<$ https://pubs.iied.org/pdfs/14639lIED.pdf> Accedido en 5 de agosto de 2019.

CANCLINI, N. G. Consumidores e cidadãos: conflitos multiculturais da globalização. Rio de Janeiro: Ed. UFRJ, p. 13-47, 1997. 
CARO, J. C.; et al. Designing a tax to discourage unhealthy food and beverage purchases: The case of Chile. Food Policy, v. 71, p. 86-100, 2017.

CARON, P.; et al. Food systems for sustainable development: proposals for a profound four-part transformation. Agronomy for sustainable development, v. 38, n. 4, p. 41, 2018.

CHANDLER, T. Missed Opportunities and Future Challenges: Globalisation, SmallFarmers and the Expanding Chilean Non-Traditional Fruit Sector, 2006.

CHILE. Ley Chile. Sobre composición nutricional de los alimentos e su publicidad. Disponible en: https://www.leychile.cl/Navegar?idLey=20606\&idVersion=2012-07-06 Accedido en 20 de septiembre 2019.

ERICKSON, P. Conceptualizing food systems for global environmental change research. Global Environmental Change, v. 18, n. 1, p. 234-245, 2008.

FREIRE, W. B.; et al. A qualitative study of consumer perceptions and use of traffic light food labelling in Ecuador. Public health nutrition, v. 20, n. 5, p. 805-813, 2017.

FRIEDMANN, H. Commentary: Food regime analysis and agrarian questions: widening the conversation. The Journal of Peasant Studies, v. 43, n. 3, p. 671-692, 2016.

HOJMAN, D. Introduction, in: Neo-liberal Agriculture in Rural Chile, [s.l.]: Springer, 1990, p. 1-20.

HOLT GIMÉNEZ, E.; SHATTUCK, A. Food crises, food regimes and food movements: rumblings of reform or tides of transformation? The Journal of peasant studies, v. 38, n. 1, p. 109-144, 2011.

LANG, T.; BARLING, D.; CARAHER, M. Food policy: integrating health, environment and society. UK: Oxford University Press, 2009.

MCMICHAEL, P. Commentary: Food regime for thought. The Journal of Peasant Studies, v. 43, n. 3, p. 648-670, 2016.

MENDOZA, C.; PINHEIRO, A.; AMIGO, H. Evolución de la situación alimentaria en Chile. Rev. chil. nutr., Santiago, v. 34, n.1, p. 62-70, marzo 2007. Disponible en https://scielo.conicyt.cl/scielo.php?script=sci_arttext\&pid=S071775182007000100007\&lng=es\&nrm=iso Accēido en 07 abr. 2020.

MONTEIRO, C. et al. Ultra-processed products are becoming dominant in the global food system. Obesity reviews, v. 14, p. 21-28, 2013.

MONTEIRO, C. et al., The UN Decade of Nutrition, the NOVA food classification and the trouble with ultra-processing, Public Health Nutrition, v. 21, n. 1, p. 5-17, 2018. 
OOSTERVEER, P.; SONNENFELD, D. A. Food, globalization and sustainability. London/New York: Routledge, 2012.

PAN AMERICAN HEALTH ORGANIZATION. Ultra-processed food and drink products in Latin America: Trends, impact on obesity, policy implications [Internet]. Washington, DC: PAHO; 2015 [2019 sept 22]. Disponible en: http://www.paho.org/hq/index.php?option=com_content\&view=article\&id=11153\&lt emid=o\&lang=es

PANEZ, A.; ROOSE, I.; FAÚNDEZ, R., Agribusiness Facing Its Limits: the Re-design of Neoliberalization Strategies in the Exporting Agriculture Sector in Chile, Land, v. 9, n. 3, p. 66, 2020

PONGUTTA, S.; et al. Packaged food consumption and understanding of front-ofpack labels in urban Thailand. Public health, v. 172, p. 8-14, 2019.

POULAIN, J. Sociologia da Obesidade. São Paulo: Senac, 2014.

PREISS, P. V.; MARQUES, F. C. Tendências no movimento de re-localização alimentar brasileiro: uma análise de Iniciativas Colaborativas de Compras. Tessituras: Revista de Antropologia e Arqueologia, v. 3, n. 2, p. 269, 2015.

RÍOS-NÚÑEZ, S. Reestructuración del sector agrario en Chile 1975-2010: entre el proteccionismo del Estado y el modelo económico neoliberal, Revista de Economia e Sociologia Rural, v. 51, n. 3, p. 515-533, 2013.

SÁNCHEZ, V. S.; SILVA, C. V. El impacto de la nueva ley de etiquetados de alimentos en la venta de productos en Chile. Revista Perfiles Económicos, n. 3, Julio, 2017.

SANTOS-ANTONIO, G.; et al. Efectos del etiquetado nutricional frontal de alimentos y bebidas: sinopsis de revisiones sistemáticas. Revista Panamericana de Salud Pública, v. 43, August, 2019.

SCHNETTLER, B.; et al. Are consumers willing to pay more for reformulated processed meat products in the context of the implementation of nutritional warnings? Case study with frankfurters in Chile. Meat science, v. 152, p. 104-108, $2019 a$.

SCHNETTLER, B., at al. How do consumers perceive reformulated foods after the implementation of nutritional warnings? Case study with frankfurters in Chile. Food quality and preference, v. 74, p. 179-188, 2019b.

SILVA, O. F.; PORTILHO, F. Sustentabilidade ambiental, consumo e cidadania. Revista Eletrônica de Geografia. São Paulo: Cortez, v. 4, n. 10, p. 255, 2005.

TREGEAR, A. Progressing knowledge in alternative and local food networks: Critical reflections and a research agenda. Journal of rural studies, v. 27, n. 4, p. 419-430, 2011. 
UAUY, R.; VIO, F., Health and nutrition transition in developing countries: the case of Chile, The Nations Nutrition. International Life Sciences Institute (ILSI),

Washington, DC. USA, p. 117-28, 2007.

UNITED NATIONS ENVIRONMENT PROGRAMME (UNEP). Food Systems and Natural Resources. A Report of the Working Group on Food Systems of the International Resource Panel. 2016.

VALVERDE-AGUILAR, M.; et al. Preferencia de etiquetado nutricional frontal: octógono frente a semáforo GDA en mercados de Lima, Perú. Acta Med, v. 35(3), p. 145-52, 2018.

WORLD BANK. Health nutrition and population statistics. 2019. Disponível em: < https://databank.worldbank.org/reports.aspx?source=311\&series=SH.STA.OWAD.ZS Acesso em: 10 ago. 2019.

Maycon Noremberg Schubert. Professor na Universidade Federal do Rio Grande do Sul (UFRGS). Área de Estudos: Consumo, Agricultura Familiar, Mercados, Alimentação. Departamento de Sociologia (UFRGS). Programa de Pós-graduação em Sociologia (PPGS/UFRGS). maycon.schubert@gmail.com

David Eduardo Ávalos. Doutorando do Programa de Pós-graduação em Sociologia (PPGS/UFRGS).david.avalosahumada@gmail.com

Como citar: SCHUBERT, Maycon Noremberg; ÁVALOS, David Eduardo. Sistemas alimentarios globales y ley de etiquetado de alimentos en Chile. Redes (St. Cruz Sul, Online), Santa Cruz do Sul, v. 25, n. 2, p. 527-544, abr. 2020. ISSN 1982-6745. Disponível em: https://online.unisc.br/seer/index.php/redes/article/view/14850 Acesso em: 15 maio 2020. doi: https://doi.org/10.17058/redes.v25i2.14850 


\section{CONTRIBUIÇÃO DOS AUTORES}

a. fundamentação teórico-conceitual e problematização - Participação de Maycon Schubert e David Ávalos

b. pesquisa de dados e análise estatística - Particição de David Ávalos

c. elaboração de figuras e tabelas - Não se aplica ao artigo

d. fotos - Não se aplica ao artigo

e. elaboração e redação do texto - Participação de David Ávalos e Maycon Schubert

f. seleção das referências bibliográficas - Participação de Maycon Schubert e David Ávalos

Fontes de financiamento - Não se aplica ao artigo 